\title{
ASO Author Reflections: The "New Normal" in Cancer Clinical Trials in the Post-Pandemic Era
}

\author{
Akhil Chawla, MD ${ }^{1}$, Toan T. Nguyen, MD, FACS ${ }^{2}$, Rebecca A. Snyder, MD, MPH, FACS ${ }^{3}$, and \\ Judy C. Boughey, MD, FACS ${ }^{4}$ \\ ${ }^{1}$ Division of Surgical Oncology, Department of Surgery, Northwestern Medicine Regional Medical Group, Northwestern \\ University Feinberg School of Medicine, Chicago, IL; ${ }^{2}$ Lakeland Regional Health, Lakeland, FL; ${ }^{3}$ Division of Surgical \\ Oncology, East Carolina University Brody School of Medicine, Greenville, NC; ${ }^{4}$ Department of Surgery, Mayo Clinic, \\ Rochester, MN
}

\section{PAST}

Clinical trials provide the highest level of evidence to guide advances in clinical practice. However, clinical trials generally are expensive for institutions to run. Trials typically require in-person patient consent visits as well as additional travel to the treating institution for research biopsies, blood tests, and other research studies. These requirements can also lead to disparities in patient enrollment. Audits have generally required travel to institutions for review of source documentation.

\section{PRESENT}

The COVID-19 pandemic affected all facets of healthcare. Delivery of cancer care was severely affected by the suspension of preventative screening as well as delays in diagnosis and treatment throughout the United States. As advancements in cancer treatment are dependent upon clinical trials, pre-existing barriers to the conduct of clinical trials were further highlighted during the COVID-19 pandemic, including low participation rates, ongoing disparities in accrual, and persistent uncertainties regarding risks. ${ }^{1}$ Furthermore, clinical trial activations in the U.S. were down $57 \%$ during the peak of the pandemic. ${ }^{2}$

(C) Society of Surgical Oncology 2021

First Received: 19 July 2021

Accepted: 19 July 2021;

Published Online: 31 July 2021

J. C. Boughey, MD, FACS

e-mail: Boughey.judy@mayo.edu
Both the accrual and conduct of oncology clinical trials were dramatically affected by the pandemic. ${ }^{3}$ Due to the prioritization of physical distancing of both patients and personnel, support staff were reassigned or furloughed, leading to a precipitous drop in eligibility screening which contributed to a decline in trial accrual. However, there were several positive changes in clinical trial conduct, including the introduction of telehealth consenting allowing for patients to mail written forms to the primary study site, increased use of local and community facilities for laboratory tests and imaging studies, and implementation of remote auditing.

As we transition from a period of strict social and medical distancing, we now face a "new normal" of a nearly or fully open clinical trial infrastructure. As many research coordinators and staff have returned to the office, we now have learned that much of the administrative work can be done without direct patient interaction. This has led to a decrease in unnecessary in-person visits, expansion of telehealth, and other risk mitigation strategies to maintain inclusion and standards for accrual.

In addition, many institutions have developed and adopted a more powerful, and perhaps more secure, electronic consenting process that will allow for expanded access to trials and expedited patient visits.

\section{FUTURE}

In the post-COVID era and hope to see a large increase in clinical trial activations and accrual to make up for the lower than anticipated enrollment during the past year. We anticipate that some of the lessons learned regarding clinical trial activity during the COVID-19 pandemic will lead 
to a change and even improvement in clinical trial conduct, allowing for follow-up blood tests, assays, and even certain treatments to be performed outside of the primary institution. Hybrid trial designs allowing for a combination of inperson and virtual visits may also prove more feasible, cost-effective, and efficient. If these changes are implemented thoughtfully and intentionally, it may allow for improved patient access to clinical trials and mitigate disparities in clinical trial access.

DISCLOSURE Toan Nguyen - Consultant for TME (Targeted Medical Education).

\section{REFERENCES}

1. Nipp RD, Hong K, Paskett ED. Overcoming barriers to clinical trial enrollment. Am Soc Clin Oncol Educ Book. 2019;39:105-14. https://doi.org/10.1200/EDBK_243729.

2. Unger JM, Xiao H. The COVID-19 pandemic and new clinical trial activations. Trials. 2021;22(1):260. https://doi.org/10.1186/s 13063-021-05219-3.

3. Boughey J, Snyder R, Kantor O, et al. Impact of COVID-19 pandemic on cancer clinical trials. Ann Surg Oncol. 2021;1-6. h ttps://doi.org/10.1245/s10434-021-10406-2.

Publisher's Note Springer Nature remains neutral with regard to jurisdictional claims in published maps and institutional affiliations. 